\title{
Profile of Knee MRI Morphometric Risk Factors for Anterior Cruciate Ligament Tear at Dr. Soetomo General Academic Hospital
}

\author{
Dyah Maya Fitriania, Rosy Setiawati ${ }^{{ }^{*}}$, Paulus Rahardjo ${ }^{a}$, Giuseppe Guglielmi ${ }^{\mathrm{b}}$ \\ *Corresponding author, rosy-s@ fk.unair.ac.id

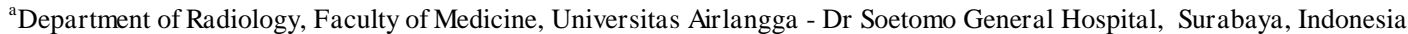 \\ bDepartment of Radiology, School of Medicine, Foggia University, Foggia, Italy
}

\begin{abstract}
Objective: Anterior Cruciate Ligament (ACL) tear can decrease quality of life especially in athlete. The physical athletic activities are external factors for ACL tear. There are also internal factor and one them is anatomical factor. Therefore, it is necessary to determine the knee morphometric risk factor profile for ACL tear.

Materials and Methods: This is a descriptive observational retrospective study with case-control design. The study data consisted of the patient's medical records and knee joint MRI study results which were collected through consecutive sampling. Morphometric variable measurements in the form of Notch Width (NW), Notch Width Index (NWI), $\alpha$-angle, Q-angle (Quadricep angle) and Medial Tibial Posterior Slope (MTPS) were assessed by two assessors. Afterwards, the mean value of each variable was determined.

Results: In ACL tear group, the mean \pm SD value for NW, NWI, Alpha angle, Q-angle and MTPS was 2.02 \pm 0.21 , $0.27 \pm 0,02,37.97 \pm 4.69,11.06 \pm 3.21$, and $9.24 \pm 2.73$, respectively, while in non-ACL tear group the mean \pm SD value was $1.97 \pm 0.23,0.29 \pm 0.02,35.59 \pm 4.52,12.03 \pm 4.08$ and $7.70 \pm 3.53$, respectively.

Conclusion: The mean NWI and Q-angle values in ACL tear group were smaller than in non-ACL tear group. The mean NW, Alpha angle, and MTPS values in ACL group were greater than in non-ACL tear group.
\end{abstract}

Keywords: Anterior Cruciate Ligament (ACL) Tear, Risk Factor, Knee Joint Morphometric, MRI

\section{Introduction}

Anterior Cruciate Ligament (ACL) tear is one of the most common knee ligament abnormalities found in athletes, its incidence ranges from 29-38 per 100,000 people. ${ }^{1-3}$ As a result, in the short term it might cause instability and in the long term it might cause degenerative process of knee joint. The most common type of ACL injury mechanism is non-contact type, due to pivoting movement, when the knee is slightly flexed and in valgus position then the femur undergoes external rotation as in foot maneuver movement during ball dribbling. ${ }^{4-8}$ Risk factors for ACL tears are divided into internal factors (anatomical, hormonal, neuromuscular and genetic factors) and external factors (exercise type, footwear, environment). Identification of these risk factors in athletes will be useful to adjust the type of training for each individual according to their anatomical variation for minimizing ACL tear incidence. ${ }^{1,7,-14}$ Previous study reported that anatomical factors that play some roles in ACL tear were femurotibial alignment, narrow intercondylar notch, type A stenotic notch, medial tibial plate depth, and steep lateral tibial slope. However, various measurement techniques and methods of these anatomical factors are still debatable. ${ }^{1,2,9-11,13,15-17,18}$ This study aims to provide an overview of anatomical risk factor characteristics in knee joint MRI study. 


\section{Materials and Methods}

This is a descriptive retrospective observational study with case-control design conducted at Dr. Soetomo General Academic Hospital, Surabaya, Indonesia, from July 2018 to January 2021. Inclusion criteria were knee MRI study results in patients with clinical knee injury, knee pain and suspected ligament tear, aged 10 to 50 years with accessible medical records. Patients with tibial plate or femur deformities, suboptimal positioning, or incomplete data were excluded. There were 5 variables in this study, which were NW, NWI, Alpha-angle, Q-angle and MTPS. These variables were assessed by two musculoskeletal radiologists with more than 10 years of experience. The results from the two assessors then averaged to determine the mean value for each variable.

The NWI is a ratio of bicondylar width to notch width as measured on coronal PD sequence of MRI at the intersection level between Posterior Cruciate Ligament (PCL) and ACL or at the level of popliteal sulcus of lateral femoral condyle ${ }^{3}$. As shown in Figure 1.

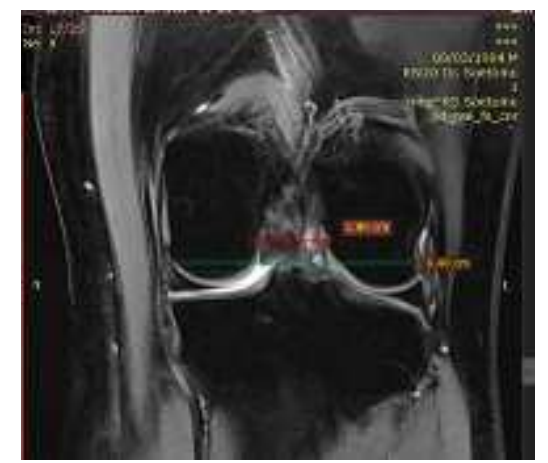

Figure 1. NW and NWI measurement illustration on coronal image. The green line is the bicondylar width $(\mathrm{BCW})$ and the red line represents $\mathrm{NW}$ calculated at the projection where the popliteal sulcus of lateral femoral condyle is visible. Notch Width Index (NWI) is obtained from NW divided by BCW. ${ }^{3}$

The Q-angle is an angle formed by the line that intersects the center of patellar bone and the center of tibial tuberosity in coronal projection with mediolateral longitudinal axis of femur. ${ }^{3}$ As showed in Figure 2.

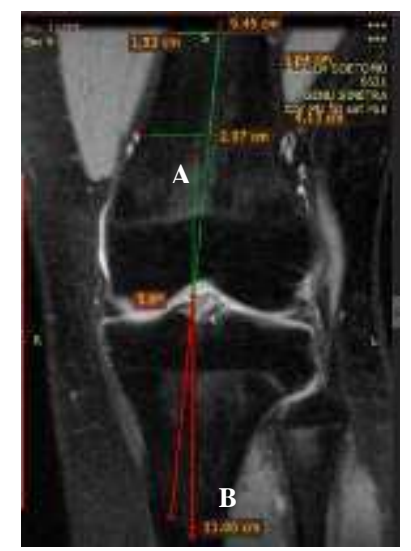

Figure 2. Quadriceps angle. The red angle is the angle formed by the intersection of the line that passes through the center of patellar bone and tibial tuberosity $\left(\mathrm{AB}\right.$ line) with mediolateral axis of femur. ${ }^{3}$ 
The $\alpha$-angle is an angle formed by the anteroposterior longitudinal axis of femur and Blumensaat line. ${ }^{3,4}$ As described in Figure 3.

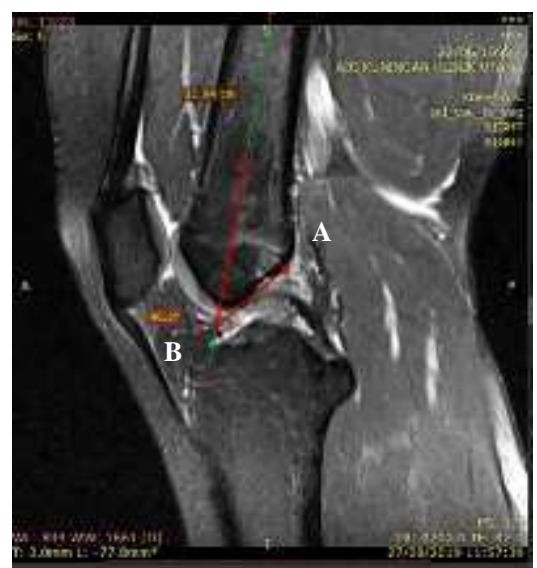

Figure 3. Illustration of $\alpha$-angle. The red angle formed by the axis of femur and the intercondylar roof or Blumensaat line (AB line) in sagittal section. ${ }^{3}$

Medial tibial posterior-inferior slope is an angle formed by a line that is perpendicular to the anteroposterior axis of tibia, with the line passes through the medial side of tibial plate at the highest point of condyles in sagittal section. ${ }^{3}$ As ilustrated in Figure 4.

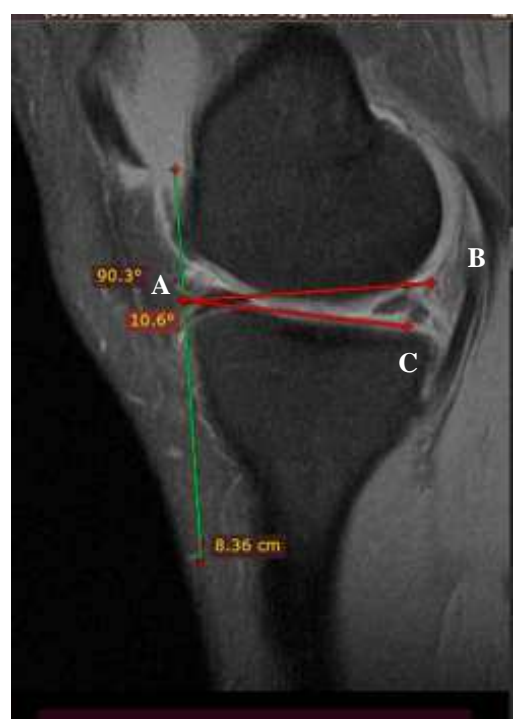

Figure 4. Medial Tibial Posterior Slope. The angle formed by a line perpendicular to the axis of tibia (AB line) and the medial tibial slope (AC line) ${ }^{3}$ 


\section{Results}

The study samples consist of 82 patients, which were divided into case (ACL tear patients) and control group (non-ACL tear patients) with equal number of patients. The interobserver suitability test was conducted for NW, NWI, Alpha angle, Q angle, and MTPS, where the kappa values for each varibles was 0.976 means very strong, 0.752 means strong, 0.854 means strong, 0.830 means strong and 0.805 means strong, respectively.

Table 1. Demographic characteristics of samples

\begin{tabular}{lrr}
\hline \multirow{2}{*}{ Characteristics } & \multicolumn{2}{c}{ ACL Tear } \\
\cline { 2 - 3 } & Yes (N=41) & No (N=41) \\
\hline Gender & $31(75.6 \%)$ & $21(51.2 \%)$ \\
Fale & $10(24.4 \%)$ & $20(48.8 \%)$ \\
Age group & & \\
$10-20$ & $14(34.1 \%)$ & $18(43.9 \%)$ \\
$21-30$ & $19(46.3 \%)$ & $15(36.6 \%)$ \\
$31-40$ & $4(9.8 \%)$ & $6(14.6 \%)$ \\
$41-50$ & $4(9.8 \%)$ & $2(4.9 \%)$ \\
\hline
\end{tabular}

In this study, it was found that ACL tear occurred more frequency in men than women with a percentage of $75.6 \%$ in the case group. Most ACL tear patients belonged to 21-30 years group. As described in Table 1.

The ACL tear group were then divided into total and partial ACL tear groups, where there were $27(66 \%)$ and $14(34 \%)$ patients, respectively. As showed in Figure 5.

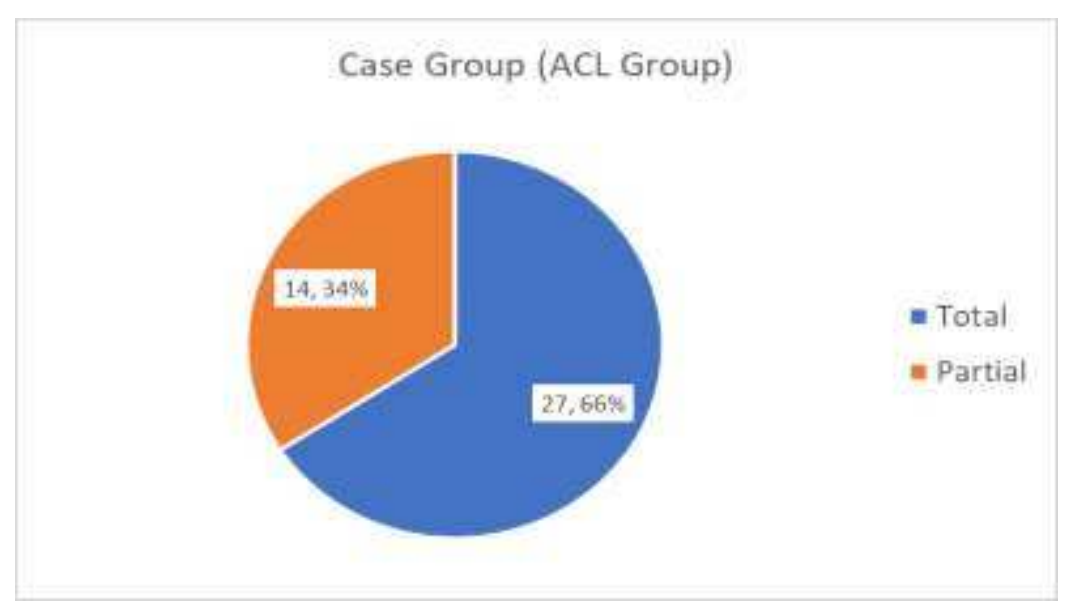

Figure 5. Diagram of total and partial ACL tear groups

The most common secondary signs in the case group were PCL buckling in total ACL tear group and bone contusion in partial ACL tear group with 26 and 3 patients, respectively. As described in Table 2. 
Table 2. Secondary sign characteristics in Partial and Total ACL tear

\begin{tabular}{lcr}
\hline \multirow{2}{*}{ Secondary Sign } & \multicolumn{2}{c}{ ACL Tear } \\
\cline { 2 - 3 } & Partial $(\mathbf{N}=14)$ & Total $(\mathbf{N}=27)$ \\
\hline Bone Contusion & 3 & 8 \\
Anterior tibial translation & 2 & 10 \\
PCL buckling & 2 & 26 \\
\hline
\end{tabular}

The mean (mean $\pm \mathrm{SD}$ ) in the case group for NW value isc $2.02 \pm 0.21$; NWI value is $0.27 \pm 0.02$; $\alpha$-angle value is 37.97 \pm 4.69 ; Q-angle is $11.06 \pm 3.21$ and MTPS value is 9.24 \pm 2.73 . The NW, $\alpha$-angle and MTPS values in the case group were higher than the control group, while the NWI and Q-angle values in the case group were smaller than the control group, as described in Table 3.

Table 3. Characteristics of morphometric variable values in ACL tear and non-ACL tear group

\begin{tabular}{lcr}
\hline \multirow{2}{*}{ Variable } & \multicolumn{2}{c}{ ACL Tear } \\
\cline { 2 - 3 } & \multicolumn{1}{c}{ Yes $(\mathbf{N}=\mathbf{4 1})$} & \multicolumn{1}{c}{ No $(\mathbf{N}=\mathbf{4 1})$} \\
Mean \pm SD & Mean \pm SD \\
\hline NW & $2.02 \pm 0.21$ & $1.97 \pm 0.23$ \\
NWI & $0,27 \pm 0.02$ & $0.29 \pm 0.02$ \\
$\alpha$-angle & $37.97 \pm 4.69$ & $35.59 \pm 4.52$ \\
Q angle & $11.06 \pm 3.21$ & $12.03 \pm 4.08$ \\
MTPS & $9.24 \pm 2.73$ & $7.70 \pm 3.53$ \\
\hline
\end{tabular}

The ACL group then was divided into two subgroups, partial and total tear group. The total tear group had lower NW, NWI, Q-angle and MTPS values than the partial group. Meanwhile, the $\alpha$-angle value was higher in the total tear group compared to the partial tear group as showed in Table 4.

Table 4. Characteristics of morphometric variable values in the total and partial ACL tear group.

\begin{tabular}{lcr}
\hline \multirow{2}{*}{ Variable } & \multicolumn{2}{c}{ Tear ACL } \\
\cline { 2 - 3 } & $\begin{array}{c}\text { Partial } \\
\text { Mean } \pm \text { SD }\end{array}$ & $\begin{array}{c}\text { Total } \\
\text { Mean } \pm \text { SD }\end{array}$ \\
\hline NW & $2.04 \pm 0.23$ & $2.00 \pm 0.20$ \\
NWI & $0.28 \pm 0.19$ & $0.27 \pm 0.02$ \\
$\alpha$-angle & $36.56 \pm 5.06$ & $38.69 \pm 4.40$ \\
Q angle & $12.26 \pm 3.69$ & $10.44 \pm 2.80$ \\
MTPS & $10.38 \pm 2.28$ & $8.65 \pm 2.79$ \\
\hline
\end{tabular}

\section{Discussion}

Knee MRI morphometric risk factors which are internal risk factors for ACL tear was studied in this research, including NW, NWI, $\alpha$-angle, Q-angle and MTPS. When an individual has a morphometric risk factor, both internal and external factor modification could be conducted as a follow-up. For internal factors, the supporting muscles of the knee joint could be strengthened, one of which is the quadriceps muscle, therefore the knee will become more stable and the ACL load will be reduced. The modifiable external factor 
is the reduction of movements that have a high risk of causing ACL tears such as pivoting, jumping movements, and sudden stops. Training that is devoted for good and correct technique in performing these movements will also help prevent ACL tears. ${ }^{19,20}$

In this study, it was found that ACL tear was found more in men than women. This finding is consistent with previous studies from Prince et al and Zeh et al, which stated that in immature (young) skeletal group, ACL tear in men, avulsion fractures, and partial tears were more likely to be found. ${ }^{11,21}$ However, this finding was inconsistence with a study from Bayer et al, which stated that the risk of ACL tear was three to six times greater in women compared to men. ${ }^{10}$ This could be due to the fact that men have more external predisposing factors, and in this study the number men samples was higher compared to women samples.

The age group with the highest incidence of ACL tear was the 21-30 years age group. According to previous studies from Zeh et al and Shen et al, ACL tear incidence was most common in the $3^{\text {rd }}$ decade of life. ${ }^{11,15}$ This could be caused by high activity in this age group, both risky activities such as sports that require a lot of jumping, landing or sudden changes in position, as well as general activities but with a high risk of contact trauma. $^{8}$

The most common secondary or indirect sign found in this study was PCL buckling, which is an abnormal orientation of the PCL, for totalACLtear and bone contusion for partial ACL tear. Total or partial ACL tear will cause loosened ligaments resulting in angular abnormalities formed by the PCL. In addition, a greater force is needed for causing the tibia and femur bone to collide with each other and bone contusion to occur. ${ }^{11,15,21-23}$ Another cause is the undetermined period of time between ACL tear incidence and MRI examination, therefore when an MRI examination was performed, bone contusion finding have disappeared.

NW and NWI value was assumed to be valuable for estimating ACL volume and could indicate impingement in ACL. ${ }^{1,9-11,15,16}$ The hypothesis in a study from Bining et al stated that the smaller the intercondylar notch, the greater the force imposed on the ACL midsubstance, which will increase the risk of injury. ${ }^{24}$ In this study, mean NW for non-ACL tear group was higher ACL tear group. This was in accordance with previous studies from Shen et al and Shaw et al, where they did not find a significant difference for NW value between ACL tear and non-tear group. ${ }^{1,15}$ The NWI value in this study showed that the case group had a lower NWI value compared to the control. In a systematic review study by Bayer et al, Domzalski et al, Shaw et al, Zeh et al, Volkan et al, the NWI value was also referred to as an important factor for ACL tear evaluation.. The NWI cut off value stated in Shen et al study for NWI was 0.25. ${ }^{1,9-11,16,1818}$

The case group had a larger $\alpha$-angle value with a mean value of $37.98 \pm 4.69$ than the control group, with a mean value of $35.59 \pm 4.52$. This result was consistent with previous studies results, which found that the case group had a mean $\alpha$-angle value of more than 38.5 degrees $^{15}, 60$ degrees $^{18}$, and 70 degrees $^{25}$, which were greater than the control group. The ACL position will be flattened causing large $\alpha$-angle value, therefore it might cause greater friction in the ACL accompanied by ACL impingement from the anterior side due to intercondylar dysplasia. ${ }^{15}$

Smaller Q-angle value with a mean value of 11.06 \pm 3.2 was found in the ACL tear group than the control group, with a mean value of $12.03 \pm 4.08$. The Q-angle in ACL tear indicates the inferior extremity alignment and indicates that the valgus shape of knee joint in the case group had a smaller mean value compared to the control group. ${ }^{10,17}$ It is inconsistent with study by Bayer et a and Sac et al which found that larger Q-angle can increased dynamic valgus and high abduction loads of the knee. ${ }^{10,17}$

The case group had a lower MTPS score with a mean value of $9.24 \pm 2.73$ than the control group, with a mean value of $7.7024 \pm 3.53$. The MTPS value in this study indicates that a greater value indicate the steeper the tibia surface. This will result in a decrease in anteroposterior stability, which is characterized by an increase in femorotibial translation quantity and an increase in ACL mechanical load. This finding is consistent with a study from Han et al, Andrade et al, Zeh et al, Shen et al, Volkan et al, Huang et al, Bayer et al, Priono et al study, where they reported a significant correlation between an increase in MTPS value with ACL tear incidence. ${ }^{2,9-11,13,15,18,26}$ They also found that the case group had a mean MTPS value of more than 14.4 degrees. $^{8}$ 
Between total and partial tear groups, it was found that NW, NWI, Q-angle and MTPS values were smaller in total tear group than in partial tear group. Meanwhile, the $\alpha$-angle was found to be higher in total tear group than partial tear group. This was not in accordance with a study from Saxena et al, where they found that the inclination angle of the intercondylar roof, which is referred in this study as the $\alpha$-angle, had a lower value in total tear group compared to partial tear group. ${ }^{14}$

Limitations of this study are: first, the total number of female and male samples were not the same, with large gap. Secondly, the ACL tearing mechanism of contact and non-contact was not assessed in this study and third, the ACL tear diagnosis was not proven on all samples by arthroscopy as a gold standart, which may cause misclassification bias. Future studies with more samples and similar men and women composition are needed. Furthermore, sample selection more homogeneous population will minimize external factors. The misclassification bias of independent variables could be minimized by increasing the number of MRI assessors to increase the result accuracy.

\section{Conclusion}

The highest ACL incidence was found in men (75.6\%) in the 21-30 years age group (46.3\%). The most common type of ACL tear was total ACL tear, with 27 samples $(66 \%)$, with the most common secondary signs being PCL buckling (PCL orientation abnormality) in total ACL tear and bony contusion in partial ACL tear.

The profile of knee MRI morphometric risk factors for ACL tear at Dr. Soetomo General Academic Hospital are as follows: First, mean NWI and Q-angle values in ACL tear group were smaller than in nonACL tear group. Secondly, the mean NW, Alpha angle, and MTPS values in ACL tear group were greater than in non-ACL tear group.

\section{Ethical Clearance}

This study was conducted in accordance with the Declaration of Helsinki. This study was approved by the 100 Medical Research Ethics Committee of Dr. Soetomo General Hospital, Surabaya. All participants included had given their written informed consent to participate in this study during admission.

\section{Acknowledgement}

The authors would like to thank Faculty of Medicine, Universitas Airlangga, Dr. Soetomo General Hospital and Radiology Department for supporting this study

\section{Source of Funding}

Self-Funded

\section{Conflict of Interest}

Nil

$\begin{array}{ll}\text { Abbreviations : } & \\ \text { ACL } & \text { : Anterior Cruciate Ligament } \\ \text { PCL } & \text { : Posterior Cruciate Ligament } \\ \text { NW } & \text { : Notch Width } \\ \text { NWI } & \text { : Notch Width Index } \\ \alpha \text {-angle } & \text { : Alpha angle } \\ \text { Q-angle } & \text { : Quadricep angle } \\ \text { MTPS } & \text { : Medial Tibial Posterior Slope } \\ \text { MRI } & \text { : Magnetic Resonance Imaging }\end{array}$


$\begin{array}{ll}\text { PD sequence } & \text { : Proton Density sequence } \\ \text { BCW } & \text { : Bicondylar width }\end{array}$

\section{References}

1. Shaw KA, Dunoski B, Mardis N, Pacicca D. Knee morphometric risk factors for acute anterior cruciate ligament injury in skeletally immature patients. J Child Orthop. 2015;9(2):161-168. doi:10.1007/s11832-015-0652-1

2. Priono BH, Utoyo GA, Ismiarto YD. Relationship of Acl Injury With Posterior Tibial Slope, Intercondylar Notch Width Ratio, Age, and Sex. J Orthop Traumatol Surabaya. 2019;7(2):106. doi:10.20473/joints.v7i2.2018.106-113

3. Singh N. International Epidemiology of Anterior Cruciate Ligament Injuries. Published online 2018:94-96. doi:10.31031/OPROJ.2018.01.000525

4. Marieswaran M, Jain I, Garg B, Sharma V, Kalyanasundaram D. Review Article A Review on Biomechanics of Anterior Cruciate Ligament and Materials for Reconstruction. 2018;2018.

5. Kam CK, Chee DWY, Peh WCG. Magnetic Resonance Imaging of Cruciate Ligament Injuries of the Knee. Can Assoc Radiol J. 2010;61(2):80-89. doi:10.1016/j.carj.2009.11.003

6. Domnick C, Raschke MJ, Herbort M, Domnick C, Raschke MJ, Herbort M. Biomechanics of the anterior cruciate ligament: Physiology, rupture and Biomechanics of the anterior cruciate ligament : Physiology , rupture and reconstruction techniques. 2016;(February). doi:10.5312/wjo.v7.i2.82

7. Hughes G, Watkins J. A risk-factor model for anterior cruciate ligament injury. Sport Med. 2006;36(5):411-428. doi:10.2165/00007256-200636050-00004

8. Kobayashi H, Kanamura T, Koshida S, Miyashita K, Okado T. Mechanisms of the anterior cruciate ligament injury in sports activities : A. 2010;(December):669-675.

9. Andrade R, Vasta S, Sevivas N, et al. Notch morphology is a risk factor for ACL injury: a systematic review and meta-analysis. J ISAKOS Jt Disord Orthop Sport Med. 2016;1(2):70-81. doi:10.1136/jisakos-2015-000030

10. Bayer S, Meredith SJ, Wilson K, et al. Knee Morphological Risk Factors for Anterior Cruciate Ligament Injury: A Systematic Review. J Bone Jt Surg - Am Vol. 2020;102(8):703-718. doi:10.2106/JBJS.19.00535

11. Zeh OF, Guena MN, Nyamfoum MPN, et al. Anatomical Risk Factors for Rupture of the Anterior Cruciate Ligament: Magnetic Resonance Imaging Based Knee Joint Assessment. Open J Radiol. 2017;07(04):228-240. doi:10.4236/ojrad.2017.74025

12. Prodromos CC, Han Y, Rogowski J, Joyce B, Shi K. A Meta-analysis of the Incidence of Anterior Cruciate Ligament Tears as a Function of Gender, Sport, and a Knee Injury-Reduction Regimen. Arthrosc - J Arthrosc Relat Surg. 2007;23(12):1320-1325. doi:10.1016/j.arthro.2007.07.003

13. Huang M, Li Y, Guo N. Relationship between intercondylar notch angle and anterior cruciate ligament injury: a magnetic resonance imaging analysis. Published online 2019. doi:10.1177/0300060518824447

14. Smith HC, Vacek P, Johnson RJ, Slauterbeck JR, Hashemi J, Shultz S. Risk Factors for Anterior Cruciate Ligament Injury : A Review of the. 2012;4(1):69-78. doi:10.1177/1941738111428281

15. Shen L, Jin ZG, Dong QR, Li LB. Anatomical risk factors of anterior cruciate ligament injury. Chin Med J (Engl). 2018;131(24):2960-2967. doi:10.4103/0366-6999.247207

16. Domzalski M, Grzelak P, Gabos P. Risk factors for Anterior Cruciate Ligament injury in skeletally immature patients: Analysis of intercondylar notch width using Magnetic Resonance Imaging. Int Orthop. 2010;34(5):703-707. doi:10.1007/s00264-010-0987-7

17. Saç A, Taşmektepligil MY. Correlation between the Q angle and the isokinetic knee strength and muscle activity. Turkish J Phys Med Rehabil. 2018;64(4):308-313. doi:10.5606/tftrd.2018.2366

18. Kızılgöz V, Sivrioğlu AK, Ulusoy GR, Aydın H, Karayol SS, Menderes U. Analysis of the risk 
factors for anterior cruciate ligament injury: an investigation of structural tendencies. Clin Imaging. 2018;50(October 2017):20-30. doi:10.1016/j.clinimag.2017.12.004

19. Boden BP, Sheehan FT, Torg JS, Hewett TE. Non-contact ACL Injuries: Mechanisms and Risk Factors. Am Acad Orthop Surg. 2010;18(9):520-527.

20. Hurd WJ, Snyder-mackler L. KNEE INSTABILITY AFTER ACUTE ACL RUPTURE AFFECTS MOVEMENT PATTERNS DURING THE MID-STANCE PHASE OF. 2010;25(10):1369-1377. doi:10.1002/jor.20440.KNEE

21. Prince JS, Laor T, Bean JA. MRI of anterior cruciate ligament injuries and associated findings in the pediatric knee: Changes with skeletal maturation. Am J Roentgenol. 2005;185(3):756-762. doi:10.2214/ajr.185.3.01850756

22. Tsai KJ, Chiang H, Jiang CC. Magnetic resonance imaging of anterior cruciate ligament rupture. BMC Musculoskelet Disord. 2004;5:1-5. doi:10.1186/1471-2474-5-21

23. Saxena A, B R, KV R, AS D, S P. Morphometry and Magnetic Resonance Imaging of Anterior Cruciate Ligament and Measurement of Secondary Signs of Anterior Cruciate Ligamet Tear. Bratisl lek??rske List. 2012;113(1):539-543. doi:10.4149/BLL

24. Bining J, Andrews G, Forster BB. The ABCs of the anterior cruciate ligament: A primer for magnetic resonance imaging assessment of the normal, injured and surgically repaired anterior cruciate ligament. Br J Sports Med. 2009;43(11):856-862. doi:10.1136/bjsm.2008.050492

25. Fernndez-Ja É N T, López-Alcorocho JM, Rodriguez-Iñigo E, Castelln F, Hernndez JC, GuillénGarcía P. The importance of the intercondylar notch in anterior cruciate ligament tears. Orthop J Sport Med. 2015;3(8):1-6. doi:10.1177/2325967115597882

26. Han H, Oh S, Bum C, Kang CS. Anthropometric difference of the knee on MRI according to gender and age groups. Surg Radiol Anat. Published online 2015. doi:10.1007/s00276-015-1536-2 